\title{
A MAC protocol design for optical-acoustic hybrid underwater wireless sensor network
}

\author{
Zhe Chen ${ }^{1}$, Jingjing Wang ${ }^{1}$, Xinjie Wang ${ }^{2}$, and Lingwei $\mathrm{Xu}^{1}$ \\ \{ acherchen@163.com, kathy1003@163.com, xinjie1023@163.com\} \\ ${ }^{1}$ School of Information Science and Technology, Qingdao University of Science and Technology, Qingdao \\ ${ }^{2}$ Qingdao University of Technology, Qingdao
}

\begin{abstract}
To achieve fast and reliable transmission of underwater data, a new type of optical-acoustic hybrid underwater wireless sensor network (OA-UWSN) is proposed in this paper, and a new media access control (MAC) protocol with optical-acoustic (O-A) handshake is designed for the proposed network, in which the optical-acoustic request to send (RTS)/clear to send (CTS) message handshake is used to ensure the reliability of communication. OMNET++ simulation software is used to simulate OA-UWSN and MAC protocols. The results show that the channel utilization of the proposed protocol in OA-UWSN is enhanced $24 \%$ more than T-Lohi protocol, and the OA-UWSN energy consumption of the proposed protocol is far lower than acoustic communication network.
\end{abstract}

Key words: UWSN, MAC protocol, Optical-acoustic, Hybrid

\section{Introduction}

The complexity of the underwater environment makes the underwater wireless high-speed and reliable communication become a challenge ${ }^{[1]}$. Optical channels and acoustic channels have become the main communication methods for current underwater wireless communication due to their unique advantages ${ }^{[2]}$.

The structure of paper is as follows. Section 2 introduces the proposed OA-UWSN model. Section 3 proposes a new MAC protocol as O-A handshake protocol and its transmission process. Section 4 introduces channel model, and analyzes channel utilization and network energy consumption for of the proposed network model with the MAC protocol in OMNET++ software.

\section{The model of underwater optical-acoustic hybrid sensor network}

An OA-UWSN combined the characteristics of underwater acoustic and optical communication was proposed $^{[3]}$. The OA-UWSN utilizes underwater acoustic channels to achieve the positioning of nodes and control information transmission. It also utilizes underwater optical channels to achieve the high-speed transmission of data.

Different from the network mentioned in [3], this paper proposes a new-type OA-UWSN, and the topological structure of the proposed OA-UWSN is as in Fig. 1. The network includes several different type of nodes: fixed nodes, mobile nodes, sink nodes and control center. Fixed nodes only collect and transmit data, and sleep at other time slots. Mobile nodes are responsible for analyzing the surrounding environment, thereby it will minimize energy consumption and extend network life. The control center and sink nodes can install GPS to obtain the absolute position information, then the position of mobile nodes and the fixed nodes can also obtain according to the position. Sink nodes receive the data sent from mobile nodes and transmit them to control center via radio. The control center uses acoustic channels to realize the time synchronization between the mobile nodes and the fixed nodes. It also uses radio to synchronize the time with the sink nodes. The underwater nodes communicate with each others by acoustic and optical channel.

However, the existing underwater MAC protocols are only designed for acoustic or optical network respectively. They are unsuitable for OA-UWSN. So this paper puts forward an O-A handshake MAC protocol 
suitable for the network. Each node in the network adopts O-A handshake protocol to guarantee high-speed and reliable data transmission.

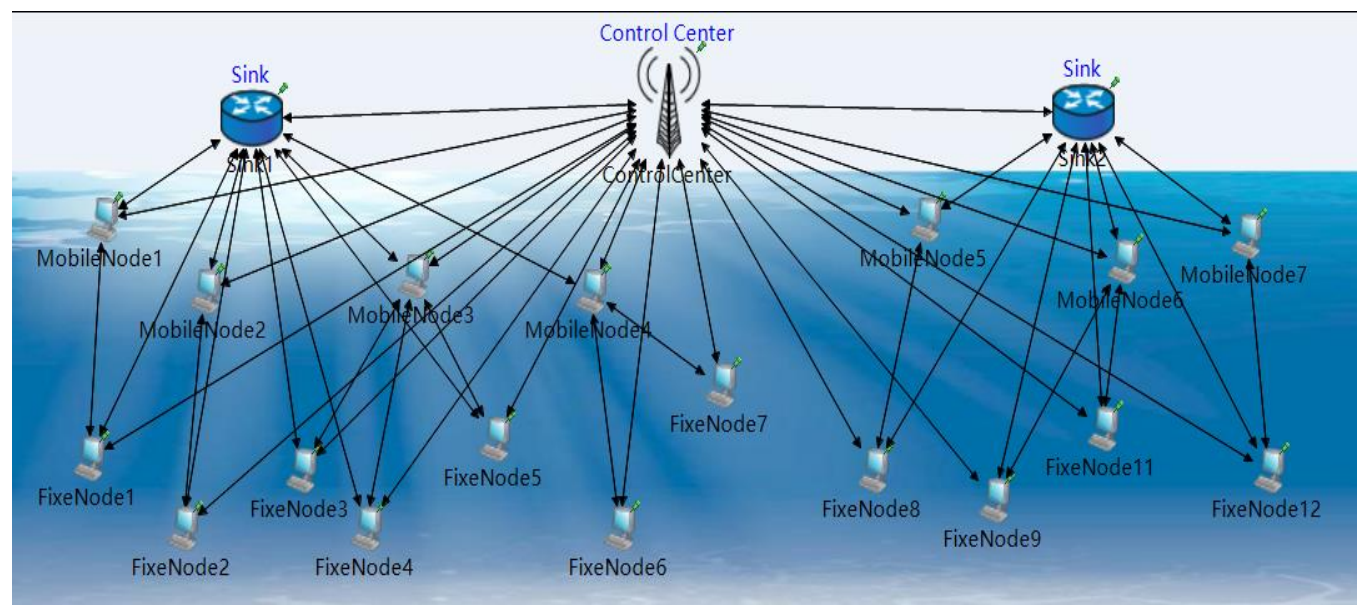

Fig. 1. OA-UWSN topology structure

\section{The MAC protocol design}

\subsection{O-A handshake protocol frame design}

This paper proposes a channel reservation MAC protocol based on O-A handshake for OA-UWSN. The protocol adopts a time-slot reservation mechanism based on the optical-acoustic handshake and back-off mechanism to avoid data collision. It divides time into frames and sets these frames as the transmission periods. And its length can be set as constant or variable values. Frame structure is shown in Fig. 2.

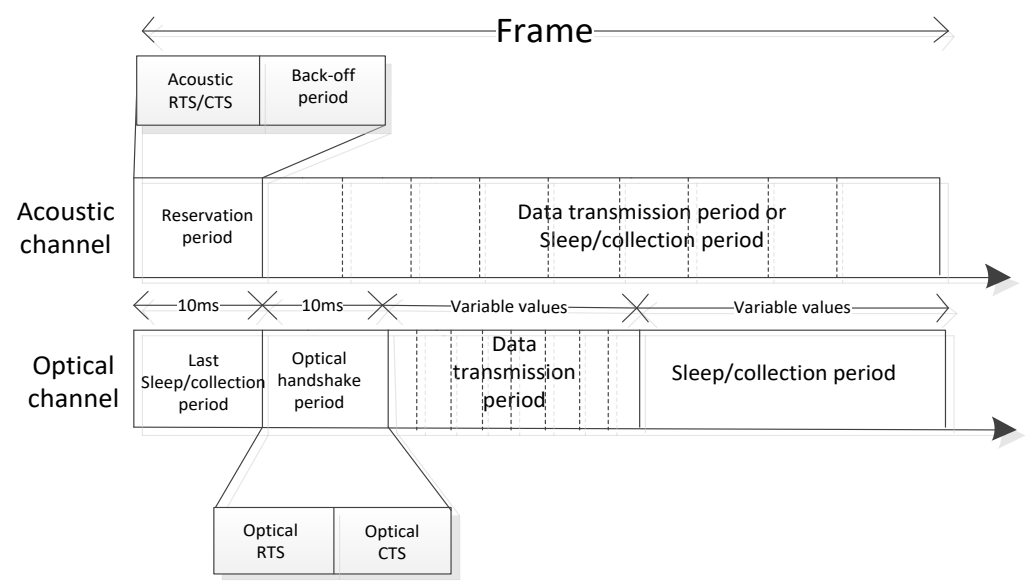

Fig. 2. Frame structure

The protocol divides frames into different periods: reservation period, optical handshake period, data transmission period and sleep/collection period. Nodes send control information via acoustic channels in reservation period and the control information includes RTS and CTS, and they also use back-off mechanism to compete channel in reservation period. Nodes send optical control information via optical channels in the acoustic handshake period. The length of the data transmission period is a variable value allocated according to the number of users. And nodes come into sleep mode or collect data in sleep/collection period. 
If the surrounding environment does not allow transmit data via optical channels, acoustic channels will be used, furtherly if acoustic channels can't be used due to the surrounding environment, nodes will transmit message again after a while. In another case, if the acoustic channel is busy, the node will not send data for a while. At the same time it randomly generates a back-off value and starts the back-off counter. When the channel idle time exceeds a period of time $T_{\text {idle }}$, the back-off counter is decremented by 1 . When the channel is busy again, the back-off counter will be suspended. Until the counter is reduced to zero, the node will start to send optical RTS message. Since then the node fail to send data each time, the back-off value will be doubled until the maximum back-off value. $W_{\max }$ means the maximum back-off value, $W_{0}$ is the initial back-off value, $W_{i}$ is the back-off value when the back-off times is $\mathrm{i}, T_{\text {idle }}$ is variable value and set to $20 \mathrm{~ms}, \mathrm{~m}$ is the maximum back-off times. The relationship between back-off value and back-off times is:

$$
\begin{cases}W_{i}=2^{i} W_{0}, & 0 \leq i \leq m \\ W_{i}=W_{\max }, & i \geq m\end{cases}
$$

Nodes conflict probability $\mathrm{P}$ is:

$$
\left\{\begin{array}{l}
P=1, f \leq g \\
P=\frac{g}{f}, f>g
\end{array}\right.
$$

Where $f$ is packet interval, $g$ is frame length, $n$ is number of nodes. Nodes back-off value $i$ is determined by $\mathrm{n} * \mathrm{P}$ and the formula 1 .

\subsection{Transmission process of O-A handshake protocol}

It's assumed that the time is synchronized in this protocol. A complete transmission process is shown in Fig. 3. The fixed node $[\boldsymbol{A}]$ and fixed node $[\boldsymbol{B}]$ send acoustic RTS messages to mobile node respectively. After the mobile node receives the RTS messages, it will send back the acoustic CTS messages. If $\boldsymbol{A}$ succeed in receiving the CTS messages, it will send optical RTS messages. Two cases, successful optical handshake and failed optical handshake, may appear later.

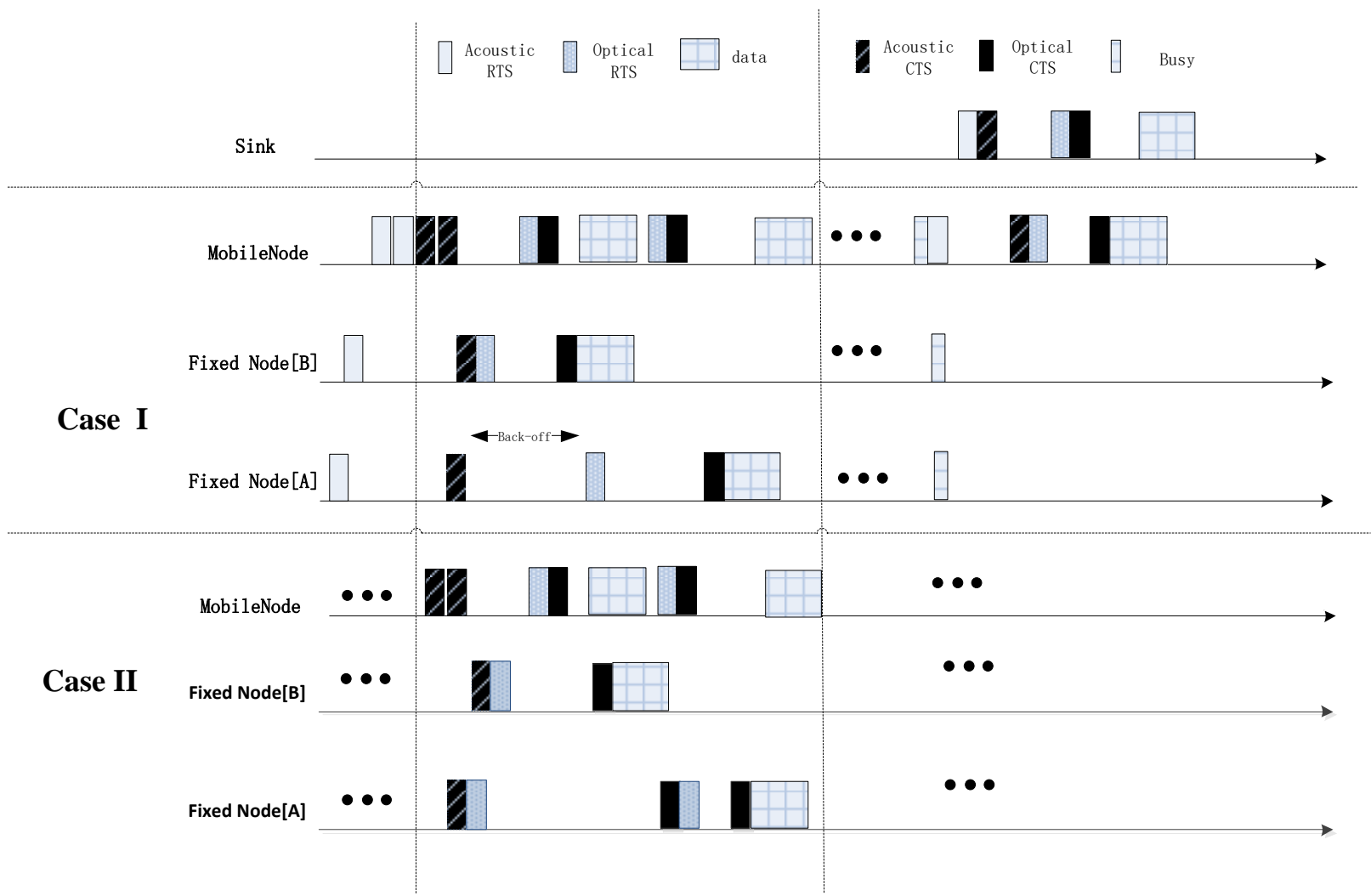

Fig. 3. A complete transmission process 
Case I: Successful optical handshake. The mobile node will make an appointment of the time to receive the data according to the sequence of the optical RTS messages, then send optical CTS messages back to the $\boldsymbol{A}$. After $\boldsymbol{A}$ receives the optical CTS messages successfully, it sends the data to the mobile node according to the reservation time slot. When the stored data of the mobile node reaches the maximum capacity, it will send "Busy" signal to the fixed nodes. After the fixed nodes receive the "Busy" signal, they will enter the sleep state. Meanwhile, the mobile node communicates with the sink node according to the same data communication rules as above. The sink node successfully receives the data and relays the data to the control center via radio, then transmission is completed.

Case II: Failed optical handshake. What is different from a successful process of data transmission is that the fixed node [A] receives the large attenuation optical CTS messages. In this condition, A estimates that optical communication is unavailable now. As the previous acoustic handshake between nodes has been successful, $\boldsymbol{A}$ will use the acoustic channel to send some important data (such as temperature-salinity-depth data, but not image and video data) and control information to the mobile node.

\section{Channel model and simulation results}

\subsection{Simulation setting parameters}

O-A UWSN uses underwater optical-acoustic hybrid mode to transmit data. The simulation experiment needs to build model for the underwater optical-acoustic channel respectively. Every node adopts underwater acoustic and optical channel model in this paper. Acoustic channel adopts the model described as in [4-5], which is for shallow-water medium-range communication and the high-frequency shallow-water warm acoustic channel. The adopted optical model is described as in [6-7], where a closed-form expression of double Gamma functions is presented to simulate the channel impulse response and a high-efficiency procedural modeling.

The simulation of underwater optical-acoustic hybrid network is completed by using OMNET++. The main setting parameters of the simulation experiment are shown in Table 1. The experiment results are concluded by taking the mean values of 150 experiment results.

Table 1. Simulation parameters

\begin{tabular}{cccc}
\hline Parameters & values & Parameters & values \\
\hline Fixed Node & 4 & Distance between nodes & $30 \mathrm{~m}$ \\
Mobile Node & 2 & Length of acoustic packet & $10 \mathrm{~kb}$ \\
Sink & 1 & Length of optical packet & $1 \mathrm{Mb}$ \\
Control Center & 1 & Acoustic sending rate & $10 \mathrm{kbps}$ \\
receiving aperture & $35 \mathrm{~mm}$ & Optical sending rat & $1 \mathrm{Mbps}$ \\
divergence angle & $0.6 \mathrm{mrad}$ & receiving efficiency & 1 \\
chlorophyll concentration & $0 \sim 1 \mathrm{mg} / \mathrm{m}^{3}$ & Phytoplankton concentration & $1 \mathrm{mg} / \mathrm{m}^{3}$ \\
Wind speed & $1 \sim 20 \mathrm{~km} / \mathrm{h}$ & Carrier frequency & $50 \mathrm{khz}$ \\
Temperature & $283 \mathrm{~K}$ & Salinity & $33 \% \mathrm{~m}$ \\
Acoustic transmission power & $1 \mathrm{w}$ & Optical transmission power & $80 \mathrm{mw}$ \\
Acoustic received power & $0.5 \mathrm{w}$ & Optical received power & $40 \mathrm{mw}$ \\
$W_{0}$ & 2 & $W_{\text {max }}$ & 128 \\
\hline
\end{tabular}

\subsection{Simulation results and analysis}

\subsubsection{Channel utilization}

The channel utilization $U$ is calculated by the ratio of transmission total time to simulation time of effective data packets in channels.

When node sends data as case I:

$$
U=\frac{T_{o d}}{T_{o d}+T_{o S}+2 *\left(T_{a l} * A R+T_{o l} * O R\right)+T_{r}+T_{C}}
$$


When the optical handshake failed as case II:

$$
U=\frac{T_{a d}}{T_{a d}+T_{a s}+2 *\left(T_{a l} * A R+T_{o l} * O R\right)+T_{r}+T_{C}}
$$

Where $T_{a d}$ is acoustic channel reception delay, $T_{\text {od }}$ is optical channel reception delay, $T_{a s}$ is acoustic channel transmission delay, $\mathrm{T}_{\mathrm{os}}$ is optical channel transmission delay, $\mathrm{T}_{\mathrm{al}}$ is acoustic channel link delay, $\mathrm{T}_{\mathrm{ol}}$ is optical channel link delay, $\mathrm{T}_{\mathrm{r}}$ is reservation period, $\mathrm{T}_{\mathrm{c}}$ is sleep or collection period tiome, OR is optical message retransmission times, $\mathrm{AR}$ is acoustic message retransmission times.

The effect of data packet transmission interval on channel utilization is shown in Fig. 4. When the sending data packet interval $f$ is very small, the receiving node has a high probability of data collision. So $i$ value becomes large and $U$ should be smalle. $U$ will reach the maximum when $n * P=1, f=2.5 \mathrm{~s}, n=2$ and $g=1.25 \mathrm{~s}$. After that, long packet interval $f$ makes $\mathrm{T}_{\mathrm{c}}$ larger and $U$ smaller, the reason is that the increase of channel idle time affects the channel utilization. The channel utilization of the T-Lohi ${ }^{[8]}$ protocol exploits high latency to detect collisions and count contenders, so it is lower than O-A handshake about channel utilization. The O-A handshake protocol can increase channel utilization by up to $24 \%$, This advantage is achieved by the O-A handshake protocol,which can avoid data collisions and take full advantage of time slots. These analysis validates that the O-A handshake protocol is more suitable for OA-UWSN.

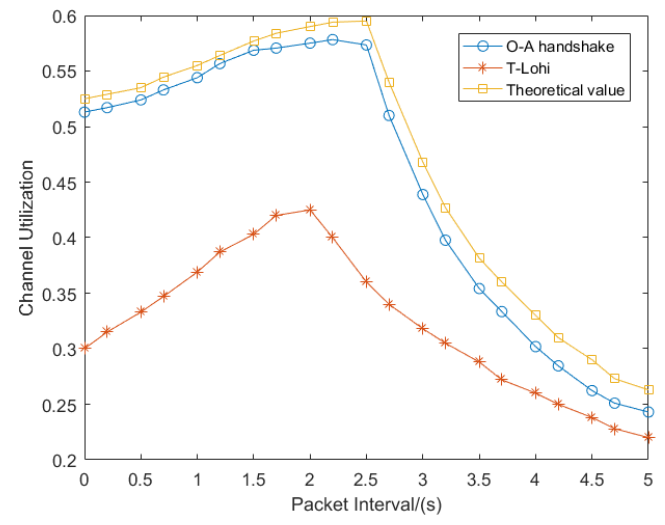

Fig. 4. Channel utilization

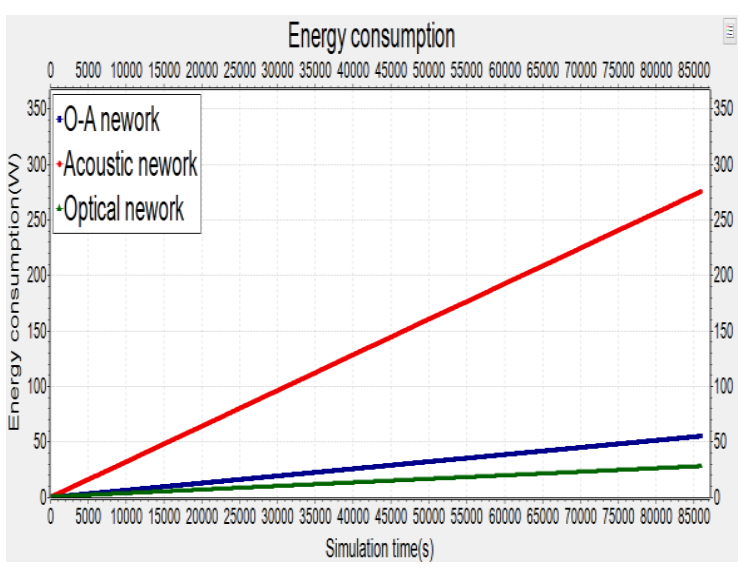

Fig. 5. Energy consumption

\subsubsection{Energy consumption analysis}

The comparison of energy consumption for different networks is shown in Fig. 5. It's seen that the energy consumption of the proposed OA-UWSN is much lower than acoustic communication network, which greatly saves the energy consumption. Although the energy consumption of OA-UWSN is slightly higher than that of optical network, the optical communication link has a great probability that data can't be transmitted when the underwater environment is poor. On the contrary, OA-UWSN can transmit important information over acoustic links, thus the reliability of the proposed OA-UWSN can be guaranteed.

\section{Summary}

A new OA-UWSN model is proposed in this pape. It utilizes underwater acoustic communication to locate the positioning of nodes, transmit control information, achieve long-distance communications and so on, and underwater optical channel is also employed as the main transmission channel to achieve high-speed data transmission. Meanwhile, the O-A handshake MAC protocol for the OA-UWSN is proposed. Finally, OMNET++ software simulation proves that the protocol can avoid the conflict effectively among nodes connection and guarantee data transmission efficiency, and the proposed OA-UWSN is more reliable when the underwater environment is poor, it also has a higher data transmission efficiency and a lower energy consumption than underwater acoustic communication network. 


\section{ACKNOWLEDGEMENTS}

This research was supported by National Natural Science Foundation of China (No. 61671261, 61771271), Key Research and Development Program of Shandong Province (No. 2016GGX101007), China Postdoctoral Science Foundation (No.2017T100490), and the University Science and Technology Planning Project of Shandong Province (No. J17KA058).

\section{References}

[1] Farizah Yunnus, Sharifah H.S. Ariffin: A Survey of Existing Medium Access Control (MAC) for Underwater Wireless Sensor Network(UWSN). Fourth Asia International Conference on Mathematical/Analytical Modelling and Computer Simulation. pp.544-549 (2010)

[2] Xianhui Che, Ian Wells: TDMA frame design for a prototype underwater RF communication network. Ad Hoc Network. 10(3):317-327 (2012)

[3] Wang J, Shi W: Design of optical-acoustic hybrid underwater wireless sensor network. Journal of Network \& Computer Applications. 92(2017): 59-67 (2017)

[4] Weigang Bai, Haiyan Wang: Modeling Underwater Time-Varying Acoustic Channel Using OPNET. Applied Mechanics and Materials. pp. 1178-1183 (2013)

[5] Naderi M, Zajić A, Ptzäold M U: A Non-Isovelocity Geometry-Based Underwater Acoustic Channel Model. IEEE Transactions on Vehicular Technology, 99(2017):1-1(2017)

[6] Shijian Tang: Impulse Response Modeling for Underwater Wireless Optical Communication Links. IEEE Transactions on Communications. 62(1): 226-234 (2014)

[7] Oubei H M, Zedini E, et al.: Efficient Weibull channel model for salinity induced turbulent underwater wireless optical communications. Opto-Electronics and Communications Conference (OECC) and Photonics Global Conference (PGC). pp. $1-2(2017)$

[8] Affan A. Syed, Wei Ye, et al.: A New Class of MAC Protocols for Underwater Acoustic Sensor Networks. IEEE INFOCOM 2008 proceedings. pp.789-797 (2008) 